\title{
In memoriam del doctor Raúl Aceves Ortega
}

\author{
Raúl Aceves-Lozano, ${ }^{1}$ Margarita Aceves-Lozano, ${ }^{2}$ Vianca Seleste Contreras-Cordero, ${ }^{3}$ \\ Verónica Alexandra Sánchez-López, ${ }^{3}$ Tom Jilmer Castillo-Valverde, ${ }^{3}$ Clotilde Fuentes-Orozco ${ }^{3}$ y \\ Alejandro González-Ojeda ${ }^{3 *}$ \\ ${ }^{1}$ Universidad de Guadalajara, Centro Universitario de Ciencias Sociales y Humanidades, Departamento de Estudios Literarios, Jalisco; ${ }^{2 H o t e l ~ H i l t o n,}$ \\ Gerencia de Ventas, Estado de México; 'Instituto Mexicano del Seguro Social, Centro Médico Nacional de Occidente, Hospital de Especialidades, \\ Guadalajara, Jalisco. México
}

\section{Formación del académico doctor Raúl Aceves Ortega}

Sus estudios básicos los realizó en la Escuela Morelos y los terminó en el Instituto de Ciencias. Sus estudios médicos los inició en la Escuela de Medicina de la Universidad Autónoma de Guadalajara; luego pasó a la Facultad de Medicina de la Universidad Nacional Autónoma de México los cuatro años siguientes, hasta su graduación el 22 de junio de 1944. Realizó su servicio social en la población de Mexticacán, Jalisco. Después de su recepción siguió en la Ciudad de México y asistió al Servicio de Dermatología del Hospital General, Pabellón 11, donde era jefe el doctor Salvador González Herrejón, a cuyos cursos de dermatología asistió, así como a los que impartía en el mismo lugar el doctor Vicente Ramírez.

A su regreso a Guadalajara en 1946 ejerció la medicina general durante algunos meses, y luego fue invitado a colaborar en la consulta médica de los enfermos de lepra y de la piel por el doctor José Barba Rubio, jefe del Dispensario Antileproso "Doctor Salvador Garciadiego", quien ya había iniciado la construcción del Instituto Dermatológico desde principios de 1947. Inició sus actividades en el dispensario al que asistía diariamente de 9 a 13 horas en forma voluntaria y sin remuneración económica ni nombramiento oficial alguno, y de la misma manera siguió prestando sus servicios en el Instituto cuando su construcción lo permitió, interviniendo en todas las actividades de este, tanto en las de tipo asistencial como académico, docente y de investigación.
En 1948, el doctor Aceves fue nombrado profesor adjunto de la cátedra de dermatología, cuyo titular era el doctor Manuel Castañeda. La cátedra se impartía en el tercer año de la carrera de medicina y estaba divida en dos sesiones independientes: dos veces por semana la impartía el doctor Castañeda y eran clases teóricas y tres veces a la semana la impartía el doctor Aceves y eran principalmente prácticas. El doctor Raúl Aceves estuvo impartiendo su curso en la forma indicada hasta el año 1953, pero siguió con su nombramiento adjunto hasta 1964. Colaboró estrechamente con otros médicos, como el doctor Julio Flores Navarro, quien no era dermatólogo pero sí un buen internista con bastante experiencia en leprología, y los pasantes Mario Gallardo Carbajal y Gonzalo Ramírez Ponce de León, que hacían su internado y preparaban su tesis recepcionales. Además de sus actividades docentes, el doctor Raúl Aceves apoyó al incipiente Instituto Dermatológico como especialista en la enfermedad de Hansen en sus diferentes presentaciones. Entonces, los pacientes afectados con este padecimiento eran muy comunes; incluso en nuestros días esta enfermedad no ha desaparecido del entorno médico.

En 1949 fue invitado por el doctor Ángel Leaño, secretario de la Facultad de Medicina de la Universidad Autónoma de Guadalajara, a impartir la cátedra Clínica de Dermatología en dicha facultad, invitación que aceptó; inició el curso de inmediato ese mismo año impartiéndolo diariamente por la mañana. La Facultad de Medicina tenía entonces con un pequeño hospital dedicado en gran parte a la ginecoobstetricia y no
Correspondencia:

*Alejandro González-Ojeda

E-mail: avygail5@gmail.com
Fecha de recepción: 15-07-2019

Fecha de aceptación: 20-08-2019

DOI: 10.24875/GMM.19005398
Gac Med Mex. 2019;155:565-572

Disponible en PubMed

www.gacetamedicademexico.com 
contaba con consulta externa. Ya que eran necesarios los pacientes con enfermedades de la piel para el curso de dermatología, el doctor Raúl Aceves inició la consulta externa, la cual pronto creció, y como el local resultaba insuficiente, entonces promovió y logró que se construyera un departamento de consulta externa en el Hospital "Ramón Garibay", que disponía de varios consultorios y un aula para las clases y reuniones; fue jefe del departamento de 1954 a 1956. Asimismo, el doctor Raúl Aceves Ortega fue nombrado director del Hospital "Ramón Garibay "de 1956 a 1958. Durante su gestión, el hospital aumentó su capacidad, organizó nuevos servicios y mejoró en muchos aspectos. Fue jefe del Servicio de Dermatología del citado hospital desde su fundación en 1950 hasta 1974, cuando el servicio fue trasladado al Hospital "Ángel Leaño", recién construido por la universidad, donde continuó siendo el jefe del renovado Servicio de Dermatología.

Poco tiempo después fue nombrado jefe del Departamento de Dermatología de la Facultad de Medicina, que incluía el mismo servicio hospitalario y el cargo de profesor titular de la cátedra, así como trabajo administrativo, hasta 1990, cuando el doctor Raúl Aceves se retiró de las actividades docentes y asistenciales de la Facultad de Medicina de la Universidad Autónoma de Guadalajara. También fue nombrado profesor de cursos de posgrado de dermatología en la Facultad de Medicina de la Universidad de Autónoma de Guadalajara desde su fundación en 1975 hasta 1990; antes de 1975 este tipo de enseñanza no estaba reglamentada y se hacía por tutoría.

El doctor Aceves Ortega fue consultor de varios hospitales de Guadalajara, como el Hospital Regional del Pacífico de 1951 a 1967, en la etapa en el que este centro prestaba servicios hospitalarios a los trabajadores ferrocarrileros; el Hospital Infantil de la Luz, desde su fundación en 1951; el Hospital Infantil Guadalupano de 1950 a 1966; el Hospital del Carmen, de 1953 a 1970, del cual fue director de 1958 a 1963; y el Hospital Santa María Chapalita, todos ubicados en el área conurbana de Guadalajara y Zapopan.

Participó como profesor en múltiples cursos de posgrado que impartía la Sociedad Mexicana de Dermatología en la Ciudad de México, de 1959 a 1988; fue profesor invitado de la Escuela de Medicina de la Universidad Autónoma de Nuevo León en abril de 1976, de la Facultad de Medicina de la Universidad Autónoma de Aguascalientes en 1976, de la Universidad de Michoacán en 1978 y en 1979, de la Universidad de Guanajuato en 1957, de la Universidad Autónoma de Zacatecas en 1970. A finales de la

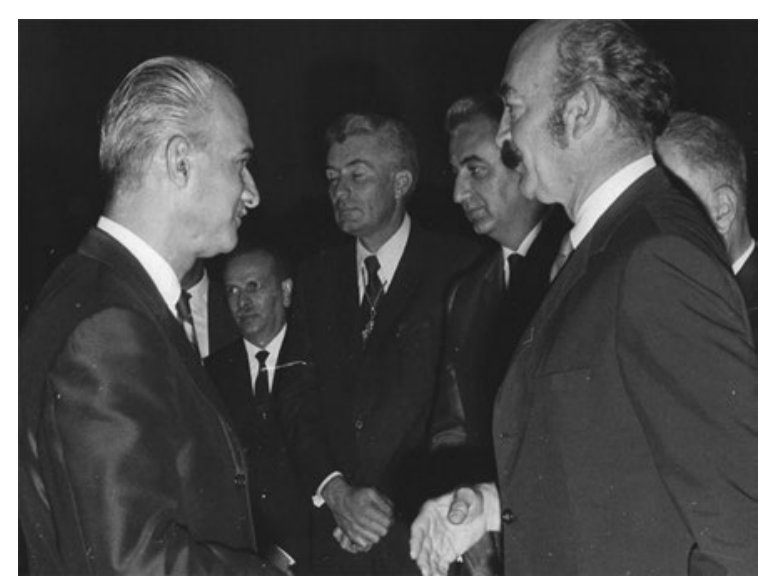

Figura 1. Ingreso del doctor Raúl Aceves Ortega a la Academia Nacional de Medicina de México. El académico doctor José Laguna García, presidente de la Academia, le dio la bienvenida al nuevo académico el 2 de septiembre de 1970.

década de 1980 organizó y dirigió simposios y seminarios de dermatología, dermatopatología y dermatología pediátrica, que resultaban de gran interés por el gran contenido clínico.

El doctor Raúl Aceves fue miembro de diversas sociedades científicas como la Sociedad Mexicana de Dermatología (1952 a 2017), miembro fundador de la Sociedad de Dermatología de Guadalajara (1984 a 2017) y de otras sociedades nacionales y extranjeras. Entre las extranjeras destacan la Real Sociedad de Medicina de Londres y la Asociación Dermatológica del Pacífico de los Estados Unidos. Ingresó a la Academia Nacional de Medicina de México el 2 de septiembre de 1970 bajo la presidencia del Académico doctor José Laguna García (Figura 1). Fue un destacado académico, con un alto sentido de la colaboración y compartió sus conocimientos y experiencias en múltiples foros organizados por nuestra academia.

Asistió a numerosos congresos nacionales y extranjeros de su especialidad, en los cuales ocupó diversos cargos y en los que intervino en la presentación de muchos trabajos. Dictó más de 200 conferencias importantes en sociedades médicas, escuelas de medicina, hospitales, cursos, mesas redondas, seminarios, simposios, reuniones, etcétera, tanto de pre como posgrado. Dirigió más de 40 tesis de pregrado y más de 20 de posgrado.

El doctor Raúl Aceves tuvo una intensa actividad científica, publicó alrededor de 150 trabajos médicos de la especialidad en revistas nacionales y extranjeras, así como varios textos de dermatología y trabajos de dermatología inéditos. En la Tabla 1 se muestran algunas publicaciones representativas del trabajo académico del doctor Aceves Ortega. Fue miembro 
Tabla 1. Trabajos representativos publicados por el académico doctor Raúl Aceves Ortega

Revista
Buletín de la Facultad de Medicina, Universidad Autónoma de
Boletín de la Facultad de Medicina, Universidad Autónoma de
Guadalajara
Boletín de la Facultad de Medicina, Universidad Autónoma de
Guadalajara
Boletín de la Facultad de Medicina, Universidad Autónoma de
Guadalajara
Boletín de la Facultad de Medicina, Universidad Autónoma de
Guadalajara
La Prensa Médica Mexicana
La Prensa Médica Mexicana

\section{La Prensa Médica Mexicana}

Revista de Medicina y Ciencias Afines

La Prensa Médica Mexicana

Dermatología, Revista Mexicana

Sonderabdruck aus der Schweizerischen Medizinischen Wochenschrift

\section{Título}

Tratamiento de la Lepra por las Sulfonas.

Transmisión de la Lepra al niño.

Tratamiento de la Lepra por las Thiosemicarbazonas.

La leprominorreacción

1952

Las tiñas.

Hidrocortisone ointment in Dermatoses.

Dermatological Service of the Hospital Ramon Garibay in

Guadalajara; comments on 1300 cases studied from 1951 to 1954.

La Prednisolona -Neomicina en el tratamiento de la Neurodermatitis diseminada y la Dermatitis por contacto.

El Vioformo en Algunas Dermatosis.

Dermatitis eczematosas.

Tratamiento de Algunas Dermatosis Piógenas con la Asociación Novobiocina Tetraciclina.

Konservative Behandlung der Nagelmykosen mit Onycho-Phytex.

Dermatosis profesional por Hidroquinona.

Tratamiento de algunas Dermatosis Piógenas con Novobicina.

La Hidrocortisona - Succinato Sódico- Intravenosa en Dermatología.

Lupus eritematoso sistémico.

La Trifluoperazina en Tratamiento de Diversas Afecciones Psicocutáneas.

Las Micosis Profundas en Nuestro Medio.

Dermatología, Mexico

La Prensa Médica Mexicana

Neo-oxylone (21-desoxy-9 alpha-fluoro-6-methylprednisolone), a new corticosteroid for topical application in some dermatoses.

Semana Médica de Mexico

Dermatitis Seborreica Infantil.

Valoración Clínica de un Nuevo Antihistamínico, Maleato de la 2-1-2 (2-Dimetilaminoetil)-3-Indetil)-Etil) Piridina, en las Dermatosis Pruriginosas.

Boletín Médico de la Universidad Autónoma de Guadalajara

Griseofulvina en Micosis Superficiales.

Griseofulvina en Micosis Profundas.

Flufenazina en Dermatología

El Vasculat en el Tratamiento del "Complejo de Pierna".

Semana Médica de Mexico

Coccidioidomicosis, Comunicación de un Caso. 
Gaceta Médica de México. 2019;155

Tabla 1. Trabajos representativos publicados por el académico doctor Raúl Aceves Ortega (Continuación)

\begin{tabular}{|c|c|c|c|}
\hline Revista & Título & Año & \\
\hline Dermatología, Revista Mexicana & Mesa Redonda sobre Corticoesteroides & 1961 & $\frac{\sigma}{6}$ \\
\hline Dermatología, Revista Mexicana & $\begin{array}{l}\text { El factor psicógeno en las dermatosis y su tratamiento } \\
\text { psicotrópico. }\end{array}$ & 1961 & $\stackrel{\frac{1}{d}}{\frac{1}{\sigma}}$ \\
\hline Sociedad Mexicana de Dermatología & $\begin{array}{l}\text { Micetomas, Análisis de } 75 \text { Casos Estudiados en la Ciudad de } \\
\text { Guadalajara. }\end{array}$ & 1961 & $\frac{5}{\square}$ \\
\hline Semana Médica de Mexico & $\begin{array}{l}\text { El Acetónido de Fluocinolona en el Tratamiento Tópico de } \\
\text { Diversas Dermatosis. }\end{array}$ & 1962 & $\dot{c}$ \\
\hline Medicina Mexicana & $\begin{array}{l}\text { New therapeutic method for psoriasis by means of the topical } \\
\text { application of fluocinolone acetonide. }\end{array}$ & 1962 & 을 \\
\hline Dermatología, Revista Mexicana & Prurigo Nodular de Hyde. & 1962 & $\frac{2}{0}$ \\
\hline Medicina Mexicana & $\begin{array}{l}\text { Fluocinolone acetonide with occlusive dressing in the topical } \\
\text { treatment of various dermatoses. }\end{array}$ & 1963 & $\frac{1}{0}$ \\
\hline Sociedad Mexicana de Dermatología. & Etiopatogenia y Tratamiento del Vitíligo, Análisis de 500 casos. & 1963 & $\frac{\sqrt{0}}{\stackrel{2}{0}}$ \\
\hline Medicina Mexicana & $\begin{array}{l}\text { Tratamiento de Algunas Formas de Dermatitis con } \\
\text { la Combinación de Acetónido de } \\
\text { Fluocinolona-ladoclorohidroxiquina. }\end{array}$ & 1964 & 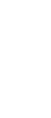 \\
\hline Medicina Mexicana & Fluocinolone acetonide and iodochlorhydroxyquin. & 1964 & $\frac{\sqrt{2}}{\frac{\pi}{\sigma}}$ \\
\hline Dermatología, Revista Mexicana & Prurito, Sintomatología, Diagnóstico y Tratamiento. & 1964 & 응 \\
\hline Memorias del II Congreso Mexicano de Dermatología. & Alopecia Areata, Estudio de 200 Casos no Seleccionados. & 1964 & 4 \\
\hline Dermatología, Revista Mexicana & Prurito, Etiopatogenia. & 1964 & $\approx$ \\
\hline La Prensa Médica Mexicana & $\begin{array}{l}\text { Nuevo Fungistático para Uso Tópico, el 3, } \\
\text { el 3,5-Dicloro-4-Fluorotiocarbanilida. }\end{array}$ & 1965 & $\begin{array}{l}\frac{u}{\partial} \\
\frac{0}{2}\end{array}$ \\
\hline La Prensa Médica Mexicana & Queilosis. Estudio de 200 casos. & 1965 & $\frac{\pi}{\sigma}$ \\
\hline Medicina Mexicana & $\begin{array}{l}\text { Clinical evaluation of } 0.01 \text { per cent solution of fluocinolone } \\
\text { acetonide in propylene glycol. }\end{array}$ & 1965 & 응 \\
\hline Sociedad Mexicana de Dermatología & Melanosis de la Cara, Estudios de 500 casos. & 1965 & оั \\
\hline Dermatología Revista Mexicana & $\begin{array}{l}\text { La pelagra en México y sus relaciones con la nutrición y } \\
\text { medicina era precortesiana. }\end{array}$ & 1967 & $\frac{\bar{d}}{\frac{\pi}{0}}$ \\
\hline Dermatología Revista Mexicana & $\begin{array}{l}\text { La pelagra en México y sus relaciones con la nutrición y } \\
\text { medicina colonial. }\end{array}$ & 1967 & 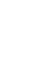 \\
\hline Dermatología Revista Mexicana & $\begin{array}{l}\text { Notas para la Historia de la pelagra sus relaciones con la } \\
\text { nutrición y la medicina en México. }\end{array}$ & 1967 & $\frac{\bar{c}}{2}$ \\
\hline Sociedad Mexicana de Dermatología & $\begin{array}{l}\text { Memorias del cuarto congreso mexicano dermatología } \\
\text { Rosácea estudio general. }\end{array}$ & 1967 & 을 - \\
\hline Sociedad Mexicana de Dermatología & Patogenia y tratamiento del acné. & 1967 & $\underline{\underline{E}}$ \\
\hline Dome Laboratorios (memorias) & Dermatitis por Drogas Comunes. & 1967 & $\stackrel{\complement}{\Perp}$ \\
\hline Dermatología Revista Mexicana & $\begin{array}{l}\text { La pelagra en México y sus relaciones con la nutrición y } \\
\text { medicina época independiente. }\end{array}$ & 1968 & $\frac{\mathrm{O}}{\mathrm{c}}$ \\
\hline Dermatología Revista Mexicana & La sífilis, diagnóstico y tratamiento. & 1968 & 은 \\
\hline Dermatología Revista Mexicana & Epidemiología de las micosis profundas. & 1969 & ᄃ \\
\hline Sociedad Mexicana de Dermatología & $\begin{array}{l}\text { Memorias del V congreso mexicano de Dermatología. } \\
\text { Epidemiología de las micosis profundas. }\end{array}$ & 1969 & 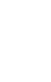 \\
\hline
\end{tabular}


Tabla 1. Trabajos representativos publicados por el académico doctor Raúl Aceves Ortega (Continuación)

\begin{tabular}{|c|c|c|}
\hline Revista & Título & Año \\
\hline Sociedad Mexicana de Dermatología & Otros usos del metrotexate y cáncer cutáneo. & 1969 \\
\hline Sociedad Mexicana de Dermatología & Esporotricosis, Nuevas observaciones clínicas. & 1969 \\
\hline Dermatología Revista Mexicana & Epidermodisplasia verruciforme. & 1974 \\
\hline Medicina Cutánea Ibero-Latino-Americana. & Dermatitis Solar. & 1974 \\
\hline International Journal of Dermatology. & Deep mycoses in Mexican children. & 1974 \\
\hline Revista Medicina & Esclerodermia localizada. & 1975 \\
\hline Revista Medicina & Etiopatogenia del acné. & 1975 \\
\hline Pediatric Dermatology. & Deep Mycosis in Children. & 1975 \\
\hline Sociedad Mexicana de Dermatología & $\begin{array}{l}\text { Memorias del VII Congreso Mexicano de Dermatología. } \\
\text { Cilindroma. }\end{array}$ & 1975 \\
\hline Sociedad Mexicana de Dermatología & Neurodermatitis atópica. & 1975 \\
\hline Sociedad Mexicana de Dermatología & Complejo de pierna, cuadros clínicos. & 1975 \\
\hline Sociedad Mexicana de Dermatología & Eritema nudoso, análisis de 74 casos. & 1975 \\
\hline Revista Medicina & Piel y Diabetes. & 1976 \\
\hline Revista Medicina & Displasia ectodérmica hidrótica. & 1977 \\
\hline Dermatología Revista Mexicana & Micetoma, análisis de 140 casos. & 1978 \\
\hline Dermatología Revista Mexicana & Pitiriasis versicolor. & 1979 \\
\hline Dermatología Revista Mexicana & Granuloma anular. & 1979 \\
\hline Dermatología Revista Mexicana & Necrobiosis Lipoidea. & 1980 \\
\hline Dermatología Revista Mexicana & Epitelioma Espinocelular análisis de 300 casos. & 1981 \\
\hline Dermatología Revista Mexicana & Pioderma gangrenosa, estudio de 12 casos. & 1981 \\
\hline Dermatología Revista Mexicana & Bacilo de la Lepra. & 1981 \\
\hline Dermatología Revista Mexicana & Las espiroquetas. & 1981 \\
\hline Dermatología Revista Mexicana & Inmuno dermatosis. & 1982 \\
\hline Dermatología Revista Mexicana & $\begin{array}{l}\text { Hiperplasias, hipertrofias y Neoplasia benignas de los tejidos } \\
\text { suaves de la cavidad oral. }\end{array}$ & 1984 \\
\hline Dermatología Revista Mexicana & Cromomicosis. & 1986 \\
\hline Actas Facultad de Medicina & Eritrodermia. & 1986 \\
\hline Actas Facultad de Medicina & Discromías. & 1987 \\
\hline Actas Facultad de Medicina & Discromías II parte. & 1987 \\
\hline Actas Facultad de Medicina & Discromías IV parte. & 1988 \\
\hline Actas Facultad de Medicina & Dermatitis Atópica primera parte. & 1988 \\
\hline Actas Facultad de Medicina & Dermatitis Atópica, Neurodermatitis diseminada parte II. & 1988 \\
\hline Actas Facultad de Medicina & Docentes en las Escuelas de Medicina & 1989 \\
\hline
\end{tabular}


Historia y Filosofía de la Medicina de 1964 a 2017, y fundador de la Sociedad Jalisciense de Historia de la Medicina (1964 a 1970).

El doctor Raúl Aceves fue miembro fundador del Consejo Mexicano de Dermatología en la Ciudad de México en 1974, del cual fue presidente de 1977 a 1979, y fue miembro activo del mismo hasta 1999, año en que pasó a la categoría de miembro honorario. Perteneció a las principales Sociedades de Dermatología de Guadalajara, la Sociedad Mexicana de Dermatología, así como la Sociedad Ibero Latino Americana, algunas de ellas como miembro fundador y fue, además, miembro de la Real Sociedad de Medicina de Inglaterra.

Perteneció a varias sociedades de estudios históricos: el Centro de Estudios Históricos "Fray Antonio Tello", de Guadalajara; La Sociedad de Estudios Alteños y la Sociedad Jalisciense de Historia de la Medicina (miembro fundador).

Dejó varios libros inéditos de historia de la medicina: Historia General de la Sifilis, Historia de la Lepra en Jalisco, La pelagra en México, La dermatología en Jalisco. Aparte de la medicina, su segunda pasión era la historia de México, particularmente de la región alteña, de donde era originario. Fruto del tiempo que dedicó a la investigación histórica fueron las siguientes publicaciones:

- Hospitales de Indios y otras Fundaciones Civiles y Religiosas en Nueva Galicia, editorial Universidad de Guadalajara, 2004

- Médicos de Guadalajara durante la época colonial, Secretaría de Cultura de Jalisco, Col Voz de la Tierra, Guadalajara, 2006.

- Primitivos pobladores indígenas e hispanos de los Altos de Jalisco, Amaroma Ediciones, Guadalajara, 2005.

- Los Aceves de los Altos de Jalisco. Amaroma Ediciones, Guadalajara, 2006.

Además de su vasto trabajo académico, docente y asistencial, desde 1947 inició su trabajo profesional privado instalando su primer consultorio en el edificio que se encuentra en la esquina de Juárez y Pavo, en el centro de Guadalajara. En 1950 se mudó al edificio Barreto y en 1960 se instaló definitivamente en su consultorio de la calle Morelos \# 1586-102 donde dio consultas hasta el 2010.

Ciertamente, resulta peculiar conocer la vida profesional del Dr. Raúl Aceves, quien transitó por las principales universidades públicas y privadas: la Universidad Nacional Autónoma de México, la Universidad Autónoma de Guadalajara y, en su faceta como historiador, en la Benemérita Universidad de Guadalajara. En nuestros días, esta movilidad no parecería extraña, sin embargo, para la época que él vivió pudiese haber sido inusual.

Finalmente, el doctor Raúl Aceves Ortega fue un digno miembro de la Academia Nacional de Medicina de México, en el Occidente del país. Logró un perfecto equilibrio entre la docencia, la investigación y la asistencia. Incluso en nuestros días, muchos colegas lo recuerdan como un médico eficaz con alto sentido del compromiso asistencial a sus pacientes. Al menos 40 generaciones de egresados de la carrera de medicina de la Universidad Autónoma de Guadalajara dan fe de su profesionalismo y apego a la educación médica. Al doctor Aceves Ortega lo recordaremos siempre por la trascendencia de sus obras.

\section{La vida en familia}

A nombre de nuestra familia Aceves Lozano, deseamos agradecer profundamente a la Academia Nacional de Medicina por su interés en hacer este homenaje a la memoria de nuestro padre, y así mismo también agradecemos la generosidad y el entusiasmo del doctor Alejandro González Ojeda y su equipo, quienes aceptaron colaborar en este homenaje, a pesar de no haber conocido en vida a nuestro padre.

Originario de Tepatitlán, Jalisco, donde nació el 21 de septiembre de 1919, desde los dos años se fue a radicar a Guadalajara junto con sus familiares. Sus padres fueron el señor Félix Aceves López y la señora Emilia Ortega Aceves; fue el mayor de nueve hermanos. El 27 de enero de 1951 se casó con Margarita Lozano Barajas, con quien tuvo siete hijos en el siguiente orden: Raúl, Sergio, Jorge Eduardo, Ricardo, Margarita, Gabriela y Alberto de Jesús. De su descendencia tuvo 12 nietos: Ana Sofía, María Inés, Regina, Santiago, Sebastián, Constanza, Igor, Diego, Rodrigo, Andrés, Alberto y Carolina, y un bisnieto, Benjamín.

Le gustaba principalmente leer obras de historia de México e historia general, así como obras literarias, novelas detectivescas, libros de arte, ensayos, biografías, revistas, etcétera. Llegó a formar una importante biblioteca que ocupaba dos salas grandes en su casa. Su segunda pasión fue la investigación y la redacción de artículos científicos y obras de tipo histórico, de las que publicó cuatro.

Otra de sus pasiones era viajar junto con su esposa, familiares y amigos, con quienes realizó viajes por México, Estados Unidos, Canadá y Europa, disfrutando especialmente su viaje a España. Particularmente tuve 
la oportunidad de hacer varios viajes con mis papás, los cuales disfrutaba mucho ya que mi padre nos explicaba sobre la historia de cada uno de esos lugares, lo cual sembró en mí el gusto también por la historia.

No era muy afecto a los deportes ni a los espectáculos, solo de joven practicó el frontenis y el boliche; disfrutaba mucho de la música, en particular de la clásica, de la cual tenía una buena colección.

Recordamos mucho el tiempo que pasaba nuestro padre con todos sus hijos los fines de semana haciendo varios paseos familiares cuando éramos muy pequeños al parque de La Primavera, diferentes parques de la ciudad, Chapala en el verano, las visitas a Tepatitlán y al rancho de mi abuelo paterno Félix Aceves y las reuniones navideñas, de cumpleaños 0 de cualquier asunto familiar que nunca llegaron a faltar. Fue un padre sumamente responsable, trabajador, estudioso, culto, de carácter fuerte, pero muy justo y dedicado a sus hijos y esposa durando su matrimonio de 66 años.

Tanto como médico y persona fue muy servicial, brindaba su apoyo como médico a quien se lo pidiera y todo esto combinándolo con otra de sus pasiones, la docencia y la investigación. Era una persona muy práctica, le gustaba hacer todo tipo de trabajos manuales y arreglos caseros, en todo lo que estuviera dentro de su alcance. Al final de su vida desarrolló otros pasatiempos: los crucigramas, los rompecabezas y la infaltable película en la televisión después de cenar.

\section{Testimonio de Raúl Aceves Lozano}

Me tocó ser el primogénito de sus hijos y llevar con mucho orgullo su mismo nombre, lo que provocaba ciertas confusiones como era de esperar, pues seguido lo felicitaban a él "por su nuevo libro de poemas" (que en realidad era mío) o me preguntaban a mí por algún tema médico. En ocasiones creían que era su "hermano menor" (por mis abundantes canas y escaso pelo). Siempre compartí con él una identidad intercambiable, de profesionista y escritor, que tampoco negaba nuestras diferencias: si a él le fascinaba la historia de México, a mí me fascinaba la literatura; si él era maestro universitario de altos vuelos y gran prestigio, yo no he pasado de ser un profesor de medio pelo; si él practicaba el oficio de la soledad en su biblioteca, yo practicaba el oficio de la sociabilidad en múltiples ambientes. Semejanzas y diferencias entre un padre y un hijo, que son muy naturales; semejanzas que me permitieron compartir con él muchos gustos (la lectura, las películas, los rompecabezas) y muchas opiniones encontradas en diversos temas y asuntos de la vida.

Él fue para mí un gran ejemplo de rectitud, de trabajo, de disciplina, de amor por el conocimiento y la cultura, de franqueza y de una actitud crítica. Fue un gran maestro, generoso, duro, fuerte, insobornable y, en ocasiones, temible. No era fácil ser su amigo ni llegar al terreno de las confidencias, porque él tenía muy claro su papel de jefe de familia y de padre, lo que lo hacía mantener siempre una distancia psicológica. Sin embargo, él espontáneamente en ocasiones se abría y se ponía a conversar de muchos temas personales, recuerdos, experiencias gozosas o desagradables, anécdotas, que narraba con mucho gusto ante su auditorio improvisado de familiares. Nunca quiso escribir sus memorias, porque franco como era, seguramente más de alguno saldría mal parado, y tampoco nunca se nos ocurrió grabar sus pláticas para tener un archivo con su voz. Lástima, porque hubiera sido muy buen recuerdo (ahora cerrando los ojos y recordándolo, puedo volver a oír su peculiar tono de voz, tan claro y firme y lleno de matices emocionales).

Me pongo a pensar en todas las conversaciones que nunca pude tener con él, en todos los viajes que nunca hice con él, en todo lo que pude haber hecho con él. Por eso pensar en él me da una enorme nostalgia, más que tristeza; me da gusto haber sido su hijo por tantos años, a pesar de todas las altas y las bajas de nuestra relación; me siento orgulloso de haber tenido un padre tan cabal y realizado, al que no le llego ni a los talones. Me siento profundamente agradecido con él y mi madre, porque me dieron este enorme y complicado regalo que es la vida, y desde el lugar o dimensión espiritual donde se hallan actualmente, no dejo de sentir su presencia protectora, amorosa, guiando mí camino, como otros ángeles de la guarda. Con amor Chato.

\section{Testimonios de algunos de sus nietos}

\section{Constanza}

Yo no les voy a contar del doctor, yo les voy a hablar del abuelito, mi tito.

Era un hombre lleno de costumbres y valores. Mi tito (como cariñosamente nos referimos a muchos de nuestros abuelos) era el hombre más guapo, sabio y amoroso del mundo, él siempre tenía una sonrisa escondida detrás de su gran y perfecto bigote blanco, siempre tenía una respuesta para cada pregunta tonta que le hacía y una historia que contar. Cuando yo era 


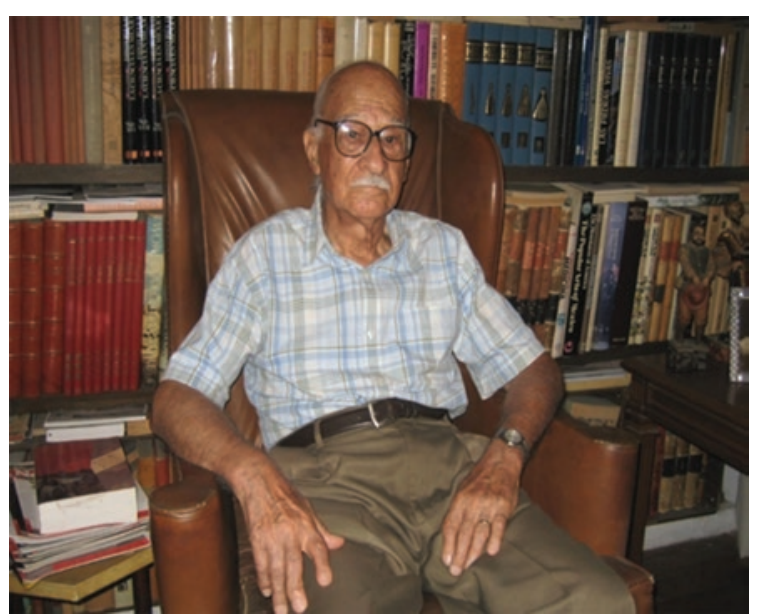

Figura 2. La biblioteca y el sillón favorito del académico doctor Raúl Aceves Ortega, quien murió en la tranquilidad de su hogar y en su lugar preferido el día 21 de mayo de 2017 a los 97 años y ocho meses.

pequeñita de cinco o seis añitos recuerdo que me subía a sus rodillas de modo de caballito, como si fuera galopando y se ponía a cantar canciones y nos contaba historias del rancho. Reíamos mucho.

Él era un hombre paciente, porque siempre estaba horas explicándome las tareas de matemáticas y no se rendía hasta que yo lo entendiera. Era un abuelito de esos que te hacía la casita de madera en el jardín abajo del árbol de limón, o nos llevaba a los primos y a mí a la tiendita a comprar todo lo que quisiéramos, nos llevaba a dar la vuelva en triciclo a la banqueta de la casa o nos llevaba a los juegos.

Alrededor de mis 32 años, le asistí en el retiro de una verruga a mi papá, yo veía que estaba feliz de que lo estuviera asistiendo y cuando terminamos me dijo que había sido la mejor asistente y me dio un beso. ¡Me sentí la nieta más feliz! ¡Hay tantas cosas lindas que recuerdo que no terminaría pronto, pero sí les puedo decir que fue un abuelito fantástico! Lleno de virtudes, dedicado a su familia y a su carrera.

\section{Julián Igor Aceves}

Recuerdo a mi abuelo Raúl (tito) como una unidad inseparable de su casa, su numerosa familia, su biblioteca con miles de libros y su jardín, el espacio favorito de mi hermana, mis primos y mío cuando éramos niños. De pequeños lo veíamos como un pequeño bosque donde podías esconderte, jugar bajo las granadas y correr alrededor de la pila (ya sin agua y con peligro de caerse en ella). Memorables son los pasillos de la casona de Avenida La Paz, testigos de que al menos en triciclo (ya que nunca aprendí a andar en bicicleta) me aventé mis buenas carreras y siempre mi abuelo viendo todo con un libro en la mano (seguramente de historia de México). Ya cansado de correr y jugar era tiempo de una jericaya, o tal vez de la nieve de vainilla que hacía mi abuela Margarita, inseparable compañera de tito, de toda la vida. Muchas veces, mientras comía, me llamaba para cantar. De él aprendí mis primeras canciones rancheras (afortunadamente conservamos documentos audiovisuales). Ahora creo que fue como un presagio, pues años más tarde trabajaría unos años en un mariachi en España, interpretando esas mismas primeras canciones, que mi abuelo me enseñó, en un ambiente de cálida vida familiar.

El 21 de mayo del 2017 murió sentado en su sillón favorito de la biblioteca (Figura 2), después de leer el periódico como era su costumbre. Dejó un legado muy importante para cada uno de sus hijos, principalmente la honradez, lealtad y el amor a su familia.

Concluimos nuestra participación en este homenaje, agradeciendo nuevamente a la Academia Nacional de Medicina de México y al doctor Alejandro González Ojeda y su grupo, por su gran apoyo para llevar a cabo la semblanza de mi padre Raúl Aceves Ortega por sus 66 años de vida académica. 